Louisiana State University

LSU Digital Commons

Faculty Publications

Department of Philosophy \& Religious Studies

2016

\title{
Cognitive Phenotypes and the Evolution of Animal Decisions
}

\author{
Tamra C. Mendelson \\ University of Maryland - Baltimore County \\ Courtney L. Fitzpatrick \\ Duke University \\ Mark E. Hauber \\ CUNY Hunter College \\ Charles H. Pence \\ Louisiana State University, cpence@lsu.edu \\ Rafael L. Rodríguez \\ University of Wisconsin-Milwaukee
}

See next page for additional authors

Follow this and additional works at: https://digitalcommons.Isu.edu/prs_pubs

Part of the Animal Studies Commons, Behavior and Ethology Commons, Biological Psychology

Commons, and the Experimental Analysis of Behavior Commons

\section{Recommended Citation}

Mendelson, T. C., Fitzpatrick, C. L., Hauber, M. E., Pence, C. H., Rodríguez, R. L., Safran, R. J., Stern, C. A., \& Stevens, J. R. (2016). Cognitive Phenotypes and the Evolution of Animal Decisions., 31 (11) Retrieved from https://digitalcommons.Isu.edu/prs_pubs/2

This Article is brought to you for free and open access by the Department of Philosophy \& Religious Studies at LSU Digital Commons. It has been accepted for inclusion in Faculty Publications by an authorized administrator of LSU Digital Commons. For more information, please contact ir@lsu.edu. 


\section{Authors}

Tamra C. Mendelson, Courtney L. Fitzpatrick, Mark E. Hauber, Charles H. Pence, Rafael L. Rodríguez, Rebecca J. Safran, Caitlin A. Stern, and Jeffrey R. Stevens 


\section{Opinion \\ Cognitive Phenotypes and the Evolution of Animal Decisions}

Tamra C. Mendelson, ${ }^{1, *, @}$ Courtney L. Fitzpatrick, ${ }^{2}$ Mark E. Hauber, ${ }^{3}$ Charles H. Pence, ${ }^{4}$ Rafael L. Rodríguez, ${ }^{5}$ Rebecca J. Safran, ${ }^{6}$ Caitlin A. Stern, ${ }^{7}$ and Jeffrey R. Stevens ${ }^{8}$

Despite the clear fitness consequences of animal decisions, the science of animal decision making in evolutionary biology is underdeveloped compared with decision science in human psychology. Specifically, the field lacks a conceptual framework that defines and describes the relevant components of a decision, leading to imprecise language and concepts. The 'judgment and decision-making' (JDM) framework in human psychology is a powerful tool for framing and understanding human decisions, and we apply it here to components of animal decisions, which we refer to as 'cognitive phenotypes'. We distinguish multiple cognitive phenotypes in the context of a JDM framework and highlight empirical approaches to characterize them as evolvable traits.

Opening the Black Box of Animal Decision Making

The fitness consequences of animal decisions (see Glossary), from mating to feeding to finding shelter, are relatively easy to characterize compared with the cognitive processes that drive them. As for any complex trait, decisions emerge from the interaction of multiple components. We consider each of these components to be a unique cognitive phenotype (Figure 1). By adopting this nomenclature, we emphasize that any component of the decision-making process can vary among individuals, have genetic and environmental components of variation, and be subject to selection. Characterizing the cognitive phenotypes that underlie animal decisions is essential for understanding how decision making evolves.

For decades, researchers have struggled to describe the internal (cognitive) components of animal decisions. During the early 20th century, behaviorists such as B.F. Skinner approached cognition as a black box, insisting that internal processes can never be inferred from observations of behavior [1]. However, modern ethologists and behavioral ecologists have made a strong case for inferring cognition from carefully designed behavioral experiments [2-5]. Moreover, as advancing technology reveals the neural architecture of cognition with unprecedented precision [6-9], we are poised to make substantive links between animal decisions in the wild, the cognitive phenotypes that produce those decisions, and the fitness consequences that shape them $[10,11]$.

Despite this progress, behavioral ecology lacks a cohesive framework for understanding the decision-making process [12,13], often leading to imprecise language and concepts when describing animal decisions in natural systems. Researchers often use the same term to refer to different cognitive phenotypes. For example, 'discrimination' might be used to refer to the perceptual ability of an animal to distinguish two similar stimuli, or it might refer to the tendency to mate with conspecifics over heterospecifics; yet, the two situations involve different cognitive and physiological processes (e.g., [7]). The absence of a cohesive decision framework is also

\section{Trends}

Animal decisions are the result of multiple interacting cognitive phenotypes.

Proximate and ultimate explanations of decisions require the characterization of cognitive phenotypes.

We provide an ontology of cognitive phenotypes in a JDM framework.

Empirical examples of successfully characterized cognitive phenotypes are highlighted.

1Department of Biological Sciences, University of Maryland Baltimore County, Baltimore, MD 21250, USA ${ }^{2}$ Department of Biology, Duke University, Durham, NC 27705, USA ${ }^{3}$ Department of Psychology, Hunter College and the Graduate Center, City University of New York, New York, NY 10065, USA

${ }^{4}$ Department of Philosophy and Religious Studies, Louisiana State University, Baton Rouge, LA 70803, USA

${ }^{5}$ Behavioral and Molecular Ecology Group, Department of Biological Sciences, University of WisconsinMilwaukee, Milwaukee, WI 53201, USA

${ }^{6}$ Department of Ecology and Evolutionary Biology, University of Colorado Boulder, Boulder, CO 80309 , USA

${ }^{7}$ Santa Fe Institute, Santa Fe, NM 87501, USA

${ }^{8}$ Department of Psychology and Center for Brain, Biology and Behavior, University of NebraskaLincoln, Lincoln, NE 68588, USA

${ }^{*}$ Correspondence: tamram@umbc.edu (T.C. Mendelson).

${ }^{\circledR}$ Twitter: @tamram 


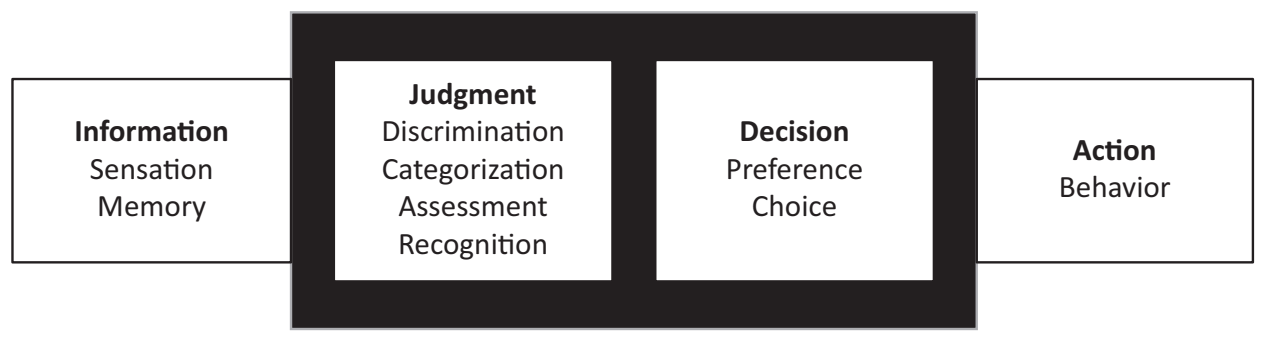

Trends in Ecology \& Evolution

Figure 1. Cognitive Phenotypes Underlying Animal Decisions. Animal behavior is initiated by information and culminates in action. The two center boxes represent the decision-making component of the process, which is modeled in psychology as a combination of judgments and decisions. Terms commonly used in behavioral ecology are mapped onto this framework and represent cognitive phenotypes that might vary among individuals and affect relative fitness. Feedback is assumed within and between all components, and components and their interactions can be affected by context, that is, the dynamic social and ecological environment in which a cognitive phenotype is expressed.

problematic when behaviors are used to infer particular cognitive phenotypes, because different cognitive processes can lead to the same behavioral output $[1,12,14]$. For example, a female might mate with a heterospecific because of any number of internal processes, including an inability to perceive a difference between conspecifics and heterospecifics or the absence of a preference (see below).

We propose that problems linking behavioral and cognitive phenotypes can be mitigated by adopting the robust conceptual framework that guides research in human decision making. This framework distinguishes two main categories of cognitive phenotypes: 'judgments' and 'decisions'. 'Judgments' refer to how individuals acquire and process information to arrive at an understanding of the situation or state of the world. 'Decisions' refer to the processes by which individuals use judgments to arrive at a course of action [15]. In other words, individuals can vary in two main ways: how they 'see' the world, and what they decide to do about it. This distinction forms the foundation of human decision-making science and frames a highly productive research agenda in human psychology [12-16].

Here, we integrate this JDM framework into behavioral ecology, assigning cognitive phenotypes such as discrimination, recognition, preference, and choice, to one of these two categories (Figure 1). This framework is a novel way for behavioral ecologists to consider animal decisions and brings multiple advantages. First, it specifies two broad classes of process with fundamentally different functions that can be modeled distinctly (e.g., Bayesian inference for judgments versus utility maximization for decisions; see below). Second, this framework emphasizes the cognitive process over the behavioral end product. These cognitive phenotypes represent the relevant axes of variation, continuous or categorical, along which individuals can differ and upon which selection can act. Linking this variation with variation in fitness serves a fundamental goal of behavioral ecology [17-19]. Third, identifying discrete cognitive phenotypes and anchoring them to a JDM framework clarifies their role in the decision-making process, which in turn allows us to identify and compare homologous processes across taxa [20] and determine which experimental approaches reveal the phenotype of interest (Boxes 1 and 2). Finally, the study of decision making is a multidisciplinary science. Integrating the JDM framework into behavioral ecology facilitates a shared understanding of concepts and techniques across the fields of human and nonhuman decision making, advancing our understanding of decision making across disciplines.

Here, we begin by describing judgment and decision making as implemented in the field of human psychology and discuss cases where it has been implicitly applied in behavioral ecology. We then identify concepts that form the core of a shared ontology, that is, a formal specification
Glossary

Assessment: extracting a measurable value of a stimulus that indicates quality or quantity.

Bayes' Rule: an algorithm for rationally updating a prior belief about the state of the world given new evidence.

Categorization: assigning two or more similar stimuli to a set and distinguishing between stimuli in different sets.

Choice: the cognitive process of selecting an action in the face of alternatives.

Cognition: the mechanisms by which animals acquire, process, store, and act on information [46]. Cognitive phenotype: a discrete cognitive feature that can be quantitatively measured and specified in terms of its neurobiology, physiology, or behavior (adapted from [47]).

Decision: an umbrella term that refers to the cognitive processes that evaluate and select options to arrive at a course of action.

Discrimination: distinguishing two or more distinct stimuli.

Judgment: making an inference or drawing a conclusion about the state of the world based on information from the internal and external environment.

Ontology: a formal specification of the concepts or entities, and the relations among those entities, comprising a particular domain of discourse.

Preference: the cognitive encoding of a ranking of options.

Recognition: responding predictably to a previously experienced and remembered stimulus. 


\begin{abstract}
Box 1. Experimental Approaches
Behavioral ecologists typically study decision making using choice tests, in which they present options to an individual that indicates choice with an action, such as moving toward an option or pulling a lever [48]. However, that action can result from multiple judgments and decisions, and a choice test cannot always distinguish among the various cognitive phenotypes that might underlie the action. If an individual fails to choose one option over another, it is unclear whether the individual failed to make the judgment (e.g. failed to discriminate) or decided not to act (e.g. lacked a preference for one stimulus over the other).
\end{abstract}

Therefore, additional information that complements a behavioral choice test is sometimes needed to specify the cognitive phenotypes underlying animal decisions. In particular, neural responses (e.g., electrophysiological recordings or magnetic resonance imaging), physiological responses (e.g., breathing rate, heart rate, or hormone levels), or behavioral responses other than choice (e.g., eye gaze or habituation-dishabituation) can be used to test for and characterize any of the cognitive phenotypes (Table I).

Discrimination can be revealed by presenting two stimuli simultaneously or sequentially and measuring neural, physiological, or behavioral responses. Different responses across stimulus types demonstrate discrimination.

To demonstrate recognition, neural, physiological, or behavioral variables should respond predictably to stimuli that have been previously experienced and differently to stimuli that have not been experienced. Recognition tests are distinguished from simple discrimination tests by determining whether subjects distinguish specifically between familiar (remembered) and novel stimuli.

For categorization, measured variables should respond both similarly to multiple exemplars of a given set of stimuli and differently to multiple exemplars not in the set.

Demonstrating assessment requires comparing the responses of measured variables with features that are known to indicate the quantity or quality of a stimulus (e.g., ornaments or odors) to determine whether and how individuals attend to those features.

Preference can be revealed by activity that exhibits a ranked response to varying stimuli.

Choice tests easily demonstrate choices by requiring an individual to choose between two or more simultaneously presented options. The neural and physiological correlates of that choice can be identified in a successful choice test and then measured in a subsequent test to demonstrate choice even if action is impeded.

Table I. Experimental Approaches to Identifying Cognitive Phenotypes ${ }^{a}$

\begin{tabular}{|c|c|c|c|}
\hline $\begin{array}{l}\text { Response } \\
\text { Variable }\end{array}$ & Techniques & $\begin{array}{l}\text { Cognitive } \\
\text { Phenotype }\end{array}$ & Refs \\
\hline \multirow[t]{3}{*}{ Neural } & Circuit visualization: in vivo imaging of active neural circuits & Discrimination & [7] \\
\hline & Electrophysiology: measures firing patterns of individual neurons & Preference & [8] \\
\hline & $\begin{array}{l}\text { Magnetic resonance imaging: measures blood flow and neural } \\
\text { activation of brain regions }\end{array}$ & Choice & [49] \\
\hline \multirow[t]{3}{*}{ Physiological } & Breathing rate: measures the rate of respiration & Discrimination & [50] \\
\hline & Heart rate: measures the rate of heart activity & Discrimination & [51] \\
\hline & $\begin{array}{l}\text { Hormone level: measures levels of hormones in blood, feces, } \\
\text { saliva, or other bodily fluids }\end{array}$ & Preference & [52] \\
\hline \multirow[t]{3}{*}{ Behavioral } & Eye gaze: measures the direction and duration of eye gaze & Assessment & [53] \\
\hline & $\begin{array}{l}\text { Habitation-dishabituation: measures when individuals can detect } \\
\text { a difference between stimuli to which they have versus have not } \\
\text { been repeatedly exposed. }\end{array}$ & Categorization & [54] \\
\hline & $\begin{array}{l}\text { Behavioral trials: measures response to options using behavioral } \\
\text { variables other than direct selection of options or association time } \\
\text { (e.g., type of display or intensity of activity) }\end{array}$ & $\begin{array}{l}\text { Discrimination, } \\
\text { categorization }\end{array}$ & [55] \\
\hline
\end{tabular}

${ }^{a} \mathrm{~A}$ host of techniques that measure neural, physiological, and behavioral responses can complement a behavioral choice test to pinpoint which components of the decision-making process contribute to a given behavioral outcome. Examples of cognitive phenotypes identified in the literature are provided, with references. 
of the concepts or entities that define an intellectual discipline and the relations among them [21]. This ontology provides more than a shared lexicon; it generates fundamental conceptual distinctions and can elucidate relations among cognitive processes that are frequently conflated in the literature. We explore common usage of these terms within behavioral ecology, provide descriptions consistent with a JDM framework, and offer alternative experimental approaches that could help identify each phenotype (Box 1), highlighting empirical examples (Box 2). Our goal is to advance a framework that facilitates an evolutionary perspective on animal decisions. A JDM view of decision making enables researchers to characterize relevant cognitive phenotypes, quantify variation in those phenotypes among individuals, and link fitness outcomes to that variation.

\section{The Judgment-Decision-Making Dichotomy}

Since the 1940s, the field of judgment and decision making has emerged from the integration of cognitive psychology, social psychology, and economics to focus on two areas. One area applies principles of psychophysics and mathematical psychology to investigate how humans make judgments about the state of the world; the other draws from economics to explore how humans make decisions [15]. Although these areas developed through different approaches and questions, they represent an important conceptual distinction between making inferences and making choices [12].

'Judgments' refer to how individuals assess information to arrive at an understanding of their world [15]. All organisms use various imperfect information (sensation, memory, etc.) to make a judgment or inference about the true state of the world or to predict future states of the world. The primary measure of judgments is accuracy, that is, the correspondence between the perceived and true state of the world. Often Bayes' rule is used to assess accuracy by

\section{Box 2. Empirical Examples of Cognitive Phenotypes}

Behavior Demonstrates Discrimination and Categorization without Preference in Sticklebacks

Kozak et al. [55] showed that male sticklebacks (Figure IA) court conspecific and heterospecific females with equivalent intensity; however, they vary the type of courtship display depending on the species, using behavior that is typical of the species being courted. This indicates an ability to discriminate the two species without a preference for one over the other. This experiment also demonstrated categorization; males exhibited the same display to multiple individuals of one set and a different display to multiple individuals of another set.

\section{Neuronal Spike Rates Demonstrate Categorization in Zebra Finches}

Hauber et al. [9] measured the spike rates of single neurons in the auditory forebrain of female zebra finches (Figure IB) in response to male songs. Higher spike rates were recorded in response to conspecific songs compared with songs of two heterospecifics; each stimulus category was represented by multiple individual exemplars. However, spike rates in response to the two heterospecifics did not differ, suggesting the categorization of conspecific song.

\section{Eye Tracking Demonstrates Assessment In Peahens}

Female peahens (Figure IC) have been shown to choose males based on the number of eyespots in their train and the length of their long 'fishtail' feather. Yorzinski et al. [53] outfitted peahens with an eye-tracking device to monitor which features of a male were attended to by females during mate choice. Females paid most attention to the eyespots on the lower segments of the train as well as the fishtail, thus empirically supporting and quantifying assessment of these features.

\section{Neuronal Spike Rates Demonstrate Preference in Gryllus bimaculatus}

Kastarakos and Hedwig [8] showed that the strength of the phonotactic response of female Gryllus bimaculatus crickets to variation in male chirp pulse duration varies continuously (Figure ID). When exposed to the same set of stimuli, activity patterns of one neuron matched the graded female phonotaxis behavior, demonstrating a cognitively encoded ranking of options (preference) that corresponded to female decisions. 

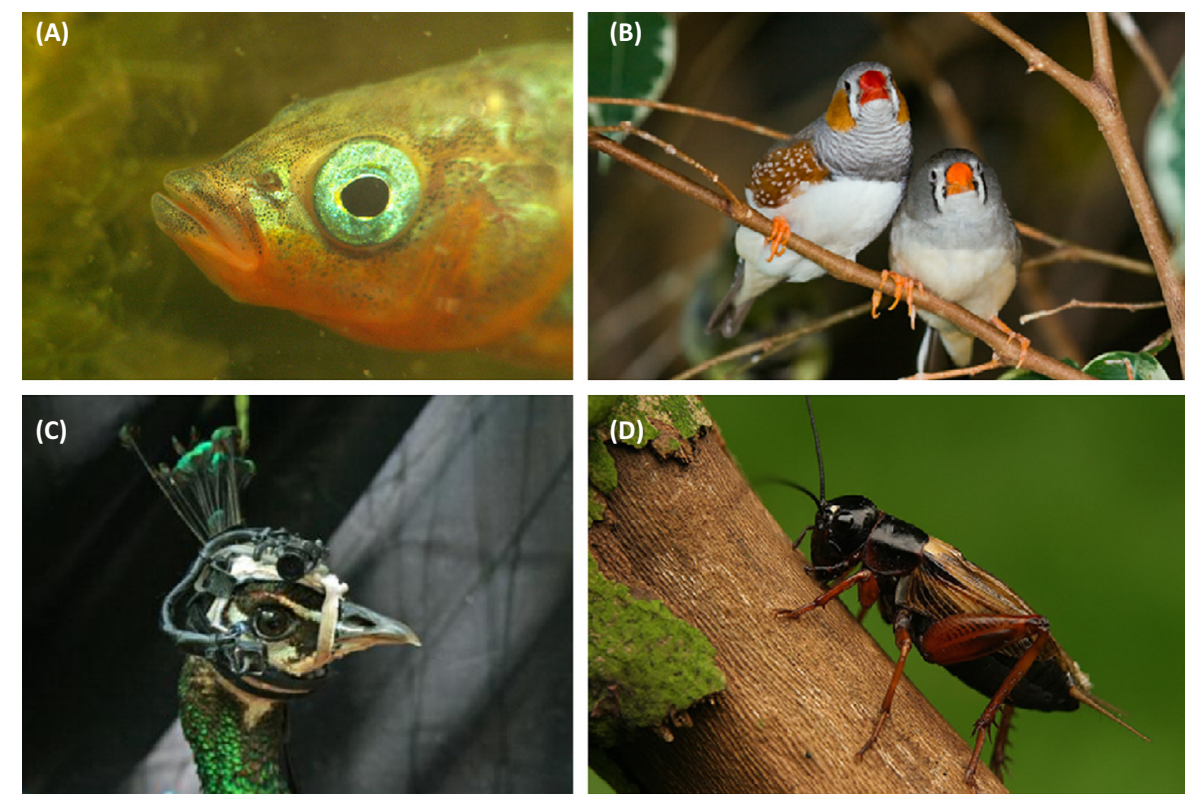

Trends in Ecology \& Evolution

Figure I. (A) Threespine stickleback Gasterosteus aculeatus (credit Piet Spaans), (B) Zebra finch Taeniopygia guttata (credit Keith Gerstung), (C) Indian peafowl Pavo cristatus (credit Jessica Yorzinksi), (D) Two-spotted cricket Gryllus bimaculatus (credit Gerd Rossen/www.digital-nature-photography.com).

determining whether an individual is accurately updating its belief about the state of the world given new information [22].

By contrast, decisions refer to how individuals determine a course of action or choose among different options [15]. Whereas judgments are typically modeled in a Bayesian framework, much of the study of decisions has come from economics and utility theory: to continue performing well in the market, an individual should choose options that offer the highest utility [23]. There is no measure of accuracy for decisions because they do not directly refer to the state of the world. The question of what is a good decision depends upon how well that decision coheres to some criterion. In economics, that criterion is expected utility maximization; in evolutionary biology, it is fitness maximization.

Although conceptually and empirically separable, judgments and decisions are nonetheless inextricably linked. Judgments are rarely made without a subsequent selection among options; decisions often require some evaluation of the state of the world; and the processes can feed back on one another [12]. For example, when a female spider encounters a male conspecific, she may categorize him as a potential mate (judgment \#1) and simultaneously or sequentially assess his quality (judgment \#2). Depending on these judgments, she may allow him to approach (decision \#1) and then mate with him (decision \#2). Early decisions can modify subsequent judgments, which in turn can modify subsequent decisions. For example, if she decides to eat the male instead of mating with him, assessment may focus on nutritional quality. Thus, as for any decision, copulation requires both judgments and decisions that can act sequentially, simultaneously, and iteratively.

We are not the first to apply this kind of framework to behavioral ecology. Blumstein and Bouskila [14] presented an 'assessment' and decision-making framework, where assessment was 
essentially equivalent to psychology's judgment. They pointed out that cognitive processes are difficult to glean from behavior alone, and that knowledge of 'informational states' (judgments) is critical (see Figure 1 in [14]). We extend the ideas of Blumstein and Bouskila [14], but rather than inventing a new framework and associated terminology, we suggest that JDM, as a productive psychological framework already in place, can serve to clarify thinking in behavioral ecology and help identify the proximate bases and ultimate consequences of animal decisions.

\section{Developing a Decision-Making Ontology}

Blumstein and Bouskila [14] demonstrated that behavioral ecologists might already implicitly distinguish between judgments and decisions. Nonetheless, many terms that describe the components of animal decisions are used inconsistently, interchangeably, and without a guiding framework in which to organize and distinguish them. To clarify terms in a way that is useful for behavioral researchers, we explored the use of six common and often interchangeably used terms from behavioral ecology: assessment, categorization, discrimination, recognition, preference, and choice. We conducted a targeted literature search to examine how these terms were defined or used in three well-studied behavioral domains: mating, foraging, and habitat selection. For each term, we searched in Web of Science' using the stem of the term (e.g. 'recogn"' or 'choice'), other terms that specified one of the three behavioral domains (e.g., 'forag*', 'mate', or 'habitat'), and the terms 'definition' or 'defined'. For each term, we identified a minimum of two articles per behavioral domain with clearly labeled definitions, as well as multiple additional articles from which definitions could be gleaned from usage. Based on this qualitative analysis, here we summarize usages across domains for each term and offer new or modified descriptions of each term that distinguish them one from another within the JDM framework.

\section{Judgment}

The concept of judgment is not widely applied in behavioral ecology. We advocate a description consistent with the psychological literature, as 'making an inference or drawing a conclusion about the state of the world based on information from the internal and external environment'. Four processes associated with judgments (discrimination, categorization, assessment, and recognition) are commonly used in behavioral ecology.

\section{Discrimination}

Discrimination is arguably the most basic cognitive process involved in a judgment. Environments (stimuli) vary along multiple dimensions, and distinguishing natural variants can be critical to the fitness of an individual. The concept of discrimination is used broadly in all three domains of behavioral ecology examined here. In some cases, it is defined as a cognitive process preceding action [24], but is more commonly defined as an action, when animals behave differently toward one stimulus versus another [25-27]. We consider discrimination, rather than an action, to be the cognitive process of 'distinguishing two or more distinct stimuli'.

\section{Categorization}

Categorization is another process used to infer the state of the world and is predicated on discrimination. Categorization is used often in the mating and foraging domains of behavioral ecology, but rarely in the context of habitat selection. Unlike discrimination, categorization is most commonly described as a cognitive process [28,29], although actions can be used to identify it. We describe categorization as 'assigning two or more similar stimuli to a set and distinguishing between stimuli in different sets'. Therefore, demonstrating categorization requires multiple test stimuli that either do or do not belong to a set of interest.

Assessment

Assessment is used across the three examined domains of behavioral ecology. Most commonly, assessment describes the acquisition of information related to the quantity or quality of a given 
stimulus, implicitly or explicitly referring to the fitness consequences of that stimulus [30,31]. Unlike discrimination or categorization, the concept of assessment applies to a single stimulus rather than two or more and is best described as 'extracting a measurable value of a stimulus that indicates quality or quantity'.

\section{Recognition}

Recognition is frequently used in the mating domain of behavioral ecology, but less so in foraging or habitat selection. In the mating literature, recognition describes a response to any member of a certain category, as in 'mate recognition' or 'kin recognition' (e.g., [32,33]). By contrast, in psychology, the concept of recognition generally emphasizes a role of memory [34,35]. To 'recognize' a stimulus is to experience and process it again, meaning that the stimulus has been previously processed. Therefore, recognition is 'responding predictably to a previously experienced and remembered stimulus'. A good example of recognition is individual recognition in paper wasps, in which individuals are remembered based on facial patterns [36]; however, many cases of mate recognition [37], competitor recognition [38], kin recognition, predator recognition, and species recognition, in which animals respond to sets of individuals rather than remembered stimuli, are better described as categorization than recognition. Framing these phenomena as categorization allows us to apply existing theories in psychology to behavioral ecology (e.g., exemplar versus prototype theories of categorization [39]) to better understand how these judgments are made.

\section{Decision}

'Decision' is an umbrella term that refers to the cognitive processes that evaluate and select options to arrive at a course of action. A decision itself is not an overt action or behavior; individuals may decide before acting. Yet, because decisions are further 'downstream' in the decision-making process (Figure 1), behavioral tests may provide more direct measures of decisions than of judgments.

\section{Preference}

Preference is used in all three behavioral ecology domains examined and typically is described as the act of selecting one or some options over others (e.g., [25,40,41]). Certainly behavioral experiments can identify preferences, but we distinguish an internal process of preference from its behavioral outcome. For example, neurons in the anterior protocerebrum of female Gryllus bimaculatus crickets fire at different rates depending on the pulse duration of male calls, with some durations producing higher firing rates than others [8] (Box 2). Therefore, this neural 'ranking' of stimuli is represented internally and independently of its behavioral outcome. We describe preference as 'the cognitive encoding of a ranking of options'.

\section{Choice}

Choice is used ubiquitously in behavioral ecology. Perhaps more than any other component of a decision-making process, choice is defined as an action, such as a behavior that restricts the potential set of mates [42], a specific type of foraging behavior that is driven by preference [43], or the probability of settling in a particular habitat [44]. However, although these actions result from a choice, an organism arrives at that choice before the motor activity typically measured in an experiment. For example, an individual may choose a mate but be prevented from mating due to the presence of a predator. Therefore, we describe choice as 'the cognitive process of selecting an action in the face of alternatives'.

Although no one example can illustrate all of these cognitive phenotypes perfectly, imagine the following case. A sample of female crickets faces a choice between a conspecific and heterospecific call in a phonotaxis trial (Figure 2), and their behavior varies. Some females approach the conspecific call, whereas others spend an equal amount of time with each call type. Multiple 


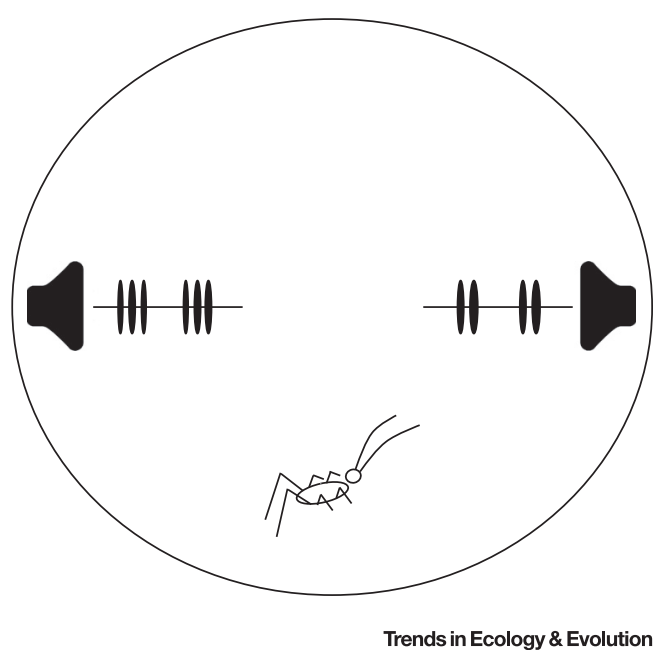

\section{Outstanding Questions}

How do cognitive phenotypes vary among individuals, genotypes, environments, etc.?

How does each cognitive phenotype influence and co-vary with others?

What are the proximate bases of cognitive phenotypes (i.e., mapping genotype to phenotype)?

To what extent does variation in cognitive phenotypes influence fitness?

What additional cognitive phenotypes contribute to animal decisions?

Figure 2. A Typical Phonotaxis Trial Offering A Female Cricket A Choice between Two Song Types.

hypotheses, targeting different cognitive phenotypes, could explain the difference in behavior, and Box 1 offers guidance on testing each of these hypotheses: (i) some females are able to discriminate between the two call types (i.e., perceive them as different), whereas other females cannot; (ii) some females recognize one call type from prior experience and others fail to remember it; (iii) some females categorize conspecific and heterospecific calls into different sets, whereas others categorize them together; and (iv) some females are able to assess the relevant features of the calls (e.g., pulse rate), whereas others cannot detect these features.

Those four hypotheses explain behavioral variation as a result of differences in judgment; however, females also can differ in decision phenotypes: (i) some females might have a preference for trait values that correspond with conspecific calls, whereas others rank conspecific and heterospecific calls equally; and (ii) all females might prefer conspecific calls, but some are motivated to make a choice, while others are not, perhaps because they recently mated.

Thus, clarifying the concepts that we use to describe and quantify animal decisions facilitates discussion and hypothesis testing within and across disciplines. Behavioral ecologists might already implicitly acknowledge the broader distinction of the JDM framework; however, by explicitly speaking a common language based on this framework, behavioral ecologists can leverage existing models (e.g., Bayesian inference or optimality) and experimental designs (e.g., habituation-dishabituation or eye tracking) from psychology to define and characterize cognitive phenotypes, test hypotheses about behavioral variation, and predict evolutionary outcomes in natural systems.

\section{Concluding Remarks and Future Directions}

With carefully designed studies that distinguish cognitive phenotypes, we can identify the genetic and neurophysiological bases of individual variation and achieve the central aim of behavioral ecology: linking the causes of animal decisions with their fitness consequences. However, whereas the fitness consequences of a decision may be relatively straightforward, its causes have been concealed inside the black box that connects information with action. The JDM framework begins to open that black box and provides an ontology of cognitive phenotypes that researchers can use to identify homologous phenotypes and characterize individual variation. However, as implemented in human psychology, the JDM framework lacks an explicit evolutionary context. Behavioral ecology provides this evolutionary perspective and can offer predictions about which judgments are likely to be subject to selection (i.e., those that affect 
fitness) and, therefore, for which we expect to observe accurate judgments [12]. Behavioral ecology also offers a clear currency of maximization for decisions (i.e., relative fitness) [45].

The core list of cognitive phenotypes discussed here presents behavioral ecologists with multiple research directions (see Outstanding Questions). As researchers with access to the broadest range of animal systems and decision-making contexts, behavioral ecologists are well positioned to identify the neural, physiological, and genetic bases of cognitive phenotypes, thus advancing our understanding of how animal decisions are generated and how decision making evolves.

\section{Acknowledgments}

This work was supported by the National Evolutionary Synthesis Center (NESCent), NSF \#EF-0905606. The authors wish to thank NESCent staff for their support and additional members of the working group 'Toward a unified evolutionary theory of decision making in animals' for helpful discussions.

\section{Resources}

i http://wokinfo.com/

\section{References}

1. Skinner, B.F. (1938) The Behavior of Organisms, AppletonCentury

2. Dukas, R. (1998) Cognitive Ecology: The Evolutionary Ecology of Information Processing and Decision Making Nol. 1), University of Chicago Press

3. Dukas, R. and Ratcliffe, J.M. (2009) Cognitive Ecology II, University of Chicago Press

4. Gould, J.L. (2004) Thinking about thinking: how Donald R. Griffin (1915-2003) remade animal behavior. Anim. Cogn. 7, 1-4

5. Griffin, D.R. and Speck, G.B. (2004) New evidence of animal consciousness. Anim. Cogn. 7, 5-18

6. Russ, B.E. et al. (2007) Neural and behavioral correlates of auditory categorization. Hearing Res. 229, 204-212

7. Clowney, E.J. et al. (2015) Multimodal chemosensory circuits controlling male courtship in drosophila. Neuron 87, 1036-1049

8. Kostarakos, K. and Hedwig, B. (2012) Calling song recognition in female crickets: temporal tuning of identified brain neurons matches behavior. J. Neurosci. 32, 9601-9612

9. Hauber, M.E. et al. (2013) Experience dependence of neural responses to different classes of male songs in the primary auditory forebrain of female songbirds. Behav. Brain Res. 243, 184-190

10. Buchanan, K.L. et al. (2013) Condition dependence, developmental plasticity, and cognition: implications for ecology and evolution. Trends Ecol. Evol. 28, 290-296

11. Thomton, A. et al. (2014) Toward wild psychometrics: linking individual cognitive differences to fitness. Behav. Ecol. 25, 1299-1301

12. Stevens, J.R. (2008) The evolutionary biology of decision making. In Better than Conscious? Decision Making, the Human Mind, and Implications for Institutions (Engel, C. and Singer, W., eds), pp. 285-304, MIT Press

13. Hammerstein, P. and Stevens, J.R. (2012) Evolution and the Mechanisms of Decision Making. MIT Press

14. Blumstein, D.T. and Bouskila, A. (1996) Assessment and decision making in animals: a mechanistic model underlying behavioral flexibility can prevent ambiguity. Oikos 77, 569-576

15. Goldstein, W.M. and Hogarth, R.M. (1997) Judgment and decision research: some historical context. In Research on Judgment and Decision Making: Currents, Connections, and Controversies (Goldstein, W.M. and Hogarth, R.M., eds), pp. 3-65, Cambridge University Press

16. Goldstein, W.M. and Hogarth, R.M., eds (1997) Research on Judgment and Decision Making: Currents, Connections, and Controversies, Cambridge University Press

17. Dall, S.R.X. et al. (2012) Variation in decision making. In Evolution and the Mechanisms of Decision Making (Hammerstein, P. and Stevens, J.R., eds), pp. 243-272, MIT Press
18. Rowe, C. and Healy, S.D. (2014) Measuring variation in cognition. Behav. Ecol. 25, 1287-1292

19. Morand-Ferron, J. and Quinn, J.L. (2015) The evolution of cognition in natural populations. Trends Cog. Sci. 19, 235-237

20. Chittka, L. et al. (2012) What is comparable in comparative cognition? Phil. Trans. Roy. Soc. B 367, 2677-2685

21. Hofweber, T. (2014) Logic and ontology. In The Stanford Encyclopedia of Philosophy (Zalta, E.N., ed.), http://plato.stanford.edu entries/logic-ontology/

22. Tenenbaum, J.B. et al. (2011) How to grow a mind: Statistics, structure, and abstraction. Science 331, 1279-1285

23. Varian, H.R. (2014) Intermediate Microeconomics: A Modern Approach. (9th edn), WW Norton \& Company

24. Niklitschek, E.J. and Secor, D.H. (2010) Experimental and field evidence of behavioural habitat selection by juvenile Atlantic $A c i$ penser oxyrinchus oxyrinchus and shortnose Acipenser brevirostrum sturgeons. J Fish Biol. 77, 1293-1308

25. Campbell, D.L.M. and Hauber, M.E. (2009) Spatial and behavioural measures of social discrimination by captive male zebra finches: implications of sexual and species differences for recognition research. Behav. Proc. 80, 90-98

26. Lackey, A.C. and Boughman, J.W. (2014) Female discrimination against heterospecific mates does not depend on mating habitat. Behav. Ecol. 25, 1256-1267

27. Herrera-Varela, M. et al. (2014) Habitat discrimination by gravid Anopheles gambiae sensu lato: a push-pull system. Malaria Journal 13, 1

28. Menzel, R. and Giurfa, M. (2001) Cognitive architecture of a minibrain: the honeybee. Trends Cog. Sci. 5, 62-71

29. Baugh, A.T. et al. (2008) Categorical perception of a natural, multivariate signal: mating call recognition in Túngara frogs. Proc. Natl. Acad. Sci. 105, 8985-8988

30. Seeley, T.D. (1989) Social foraging in honey bees: how nectar foragers assess their colony's nutritional status. Behav. Ecol. Sociobiol. 24, 181-199

31. Bonduriansky, R. (2001) The evolution of male mate choice in insects: a synthesis of ideas and evidence. Biol. Rev. 76, 305-339

32. Ryan, M.J. and Rand, A.S. (1993) Species recognition and sexual selection as a unitary problem in animal communication. Evolution $47,647-657$

33. Macedonia, J.M. et al. (2013) Species recognition of color and motion signals in Anolis grahami: evidence from responses to lizard robots. Behav. Ecol. 24, 846-852

34. Santrock, J.W. (2005) Psychology, McGraw-Hill

35. Weiten, W. (2015) Psychology: Themes and Variations, Cengage Learning 
36. Tibbetts, E.A. and Dale, J. (2007) Individual recognition: it is good to be different. Trends in Ecology \& Evolution 22, 529-537

37. Reeve, H.K. (1989) The evolution of conspecific acceptance thresholds. Am. Nat. 33, 407-435

38. Grether, G.F. (2011) The neuroecology of competitor recognition. Int. Comp. Biol. 51, 807-818

39. Ashby, F.G. and Maddox, W.T. (2005) Human category learning. Ann. Rev. Psych. 56, 149-178

40. Krivan, V. (2010) Evolutionary stability of optimal foraging: partial preferences in the diet and patch models. J. Theor. Biol. 267, 486-494

41. Hollander, F.A. et al. (2011) Maladaptive habitat selection of a migratory passerine bird in a human-modified landscape. PLOS ONE 6, e25703

42. Wiley, R.H. and Poston, J. (1996) Perspective: indirect mate choice, competition for mates, and coevolution of the sexes. Evolution 50, 1371-1381

43. Jackson, R.R. et al. (2005) A spider that feeds indirectly on vertebrate blood by choosing female mosquitoes as prey. Proc. Natl. Acad. Sci. 102, 15155-15160

44. Ravigné, V. et al. (2009) Live where you thrive: joint evolution of habitat choice and local adaptation facilitates specialization and promotes diversity. Am. Nat. 174, E141-E169

45. Kacelnik, A. (2006) Meanings of rationality. In Rational Animals? (Hurley, S. and Nudds, M., eds), pp. 87-106, Oxford University Press
6. Shettleworth, S.J. (2010) Cognition, Evolution, and Behavior, Oxford University Press

47. Santangelo, S.L. and Jagaroo, V., eds (2016) Cognitive and Behavioral Phenotypes, Springer

48. Dougherty, L.R. and Shuker, D.M. (2015) The effect of experimental design on the measurement of mate choice: a meta-analysis. Behav. Ecol. 26, 311-319

49. Voss, H.U. et al. (2010) Altered auditory BOLD response to conspecific birdsong in zebra finches with stuttered syllables. PLOS ONE 5, e14415

50. Barreto, R.E. et al. (2003) Ventilatory frequency indicates visua recognition of an allopatric predator in nalve Nile tilapia. Behav. Proc. 60, 235-239

51. Hauber, M.E. et al. (2002) Discrimination between host songs by brood parasitic brown-headed cowbirds (Molothrus ater). Anim Cogn. 5, 129-137

52. Tchernichovski, O. et al. (1998) Context determines the sex appea of male zebra finch song. Anim. Behav. 55, 1003-1010

53. Yorzinski, J.L. et al. (2013) Through their eyes: selective attention in peahens during courtship. J. Exp. Biol. 216, 3035-3046

54. Wyttenbach, R.A. et al. (1996) Categorical perception of sound frequency by crickets. Science 273, 1542-1544

55. Kozak, G.M. et al. (2009) Sex differences in mate recognition and conspecific preference in species with mutual mate choice. Evolution 63, 353-365 